\title{
A Theorem on the Complete Integral
}

\author{
By H. E. Daniels, Edinburgh University.
}

(Received 14th May, 1932. Read 4th June, 1932.)

The object of the present note is to show that a well-known theorem in the theory of non-linear partial differential equations, which is usually proved analytically, ${ }^{1}$ admits of a geometrical proof which exhibits the relations concerned in a more intuitive manner.

Theorem: Given a non-linear partial differential equation

$$
f(x, y, z, p, q)=0 .
$$

Let $f_{1}(x, y, z, p, q), f_{2}(x, y, z, p, q)$ be two independent functions satisfying

$$
\left[f_{1}, f\right]=0, \quad\left[f_{2}, f\right]=0 .
$$

Then if $p, q$ be eliminated from

$$
\begin{aligned}
& f=0 \\
& f_{1}=a_{1} \\
& f_{2}=a_{2}
\end{aligned}
$$

where $a_{1}$ and $a_{2}$ are arbitrary constants, the necessary and sufficient condition that the surface so obtained should be a complete integral of (1) is

where

$$
\left[f_{1}, f_{2}\right]=0
$$

$$
\begin{aligned}
{[F, f] } & \equiv \frac{\partial F}{\partial x} \frac{\partial f}{\partial p}+\frac{\partial F}{\partial y} \frac{\partial f}{\partial p} \\
& +\frac{\partial F}{\partial z}\left(p \frac{\partial f}{\partial p}+q \frac{\partial f}{\partial q}\right)-\frac{\partial F}{\partial p}\left(\frac{\partial f}{\partial x}+p \frac{\partial f}{\partial z}\right)-\frac{\partial F}{\partial q}\left(\frac{\partial f}{\partial y}+q \frac{\partial f}{\partial z}\right) .
\end{aligned}
$$

Solving (1) and (2) for $p$ and $q$, say

$$
\begin{aligned}
& p=\phi\left(x, y, z, a_{1}\right) \\
& q=\psi\left(x, y, z, a_{1}\right),
\end{aligned}
$$

1 See, for example, L. Bieberhach, Differenticlgleichungen (Berlin, 1923), 220-221, or Goursat, Leçons sur l'intégration des équations anx déritées partielles du premiér ordre (Paris, 1891), $167(\$ 66)$. 
and substituting in (3) we get a certain surface $S$. Now solutions of $[F, f]=0$ are, by the theory of linear partial differential equations, the same as solutions of Charpit's equations for the characteristic strips of $f=0$, viz.

$$
\frac{d x}{\frac{\partial f}{\partial p}}=\frac{d y}{\frac{\partial f}{\partial q}}=\frac{d z}{p \frac{\partial f}{\partial p}+q \frac{\partial f}{\partial q}}=\frac{d p}{-\left(\frac{\partial f}{\partial x}+p \frac{\partial f}{\partial z}\right)}=\frac{d q}{-\left(\frac{\partial f}{\partial y}+q \frac{\partial f}{\partial z}\right)} .
$$

Hence if

$$
f_{2}^{\prime}=\alpha_{2}^{\prime}
$$

were another independent integral of $[F, f]=0$, then (1), (2), (4) would give another surface $S^{\prime}$ intersecting $S$ along a characteristic curve of $f=\overrightarrow{0}$. S S must then be generated by characteristic curves $C$ of $f=0$, whose associated strips satisfy $p=\phi, q=\psi$.

We have the identity

$$
[F, f] \equiv-[f, F]
$$

so that, since $f_{1}$ satisfies $[F, f]=0, f$ satisfies $\left[F, f_{1}\right]=0$. Again, if we impose the relation

$$
\left[f_{1}, f_{2}\right]=0,
$$

then $f_{2}$ satisfies $\left[F, f_{1}\right]=0$. Also $\left[f_{1}, f_{1}\right] \equiv 0$, so $f=0, f_{1}=a_{1}, f_{2}=a_{2}$ are three integrals of Charpit's equations corresponding to the partial differential equation

$$
f_{1}=a_{1},
$$

and $S$ is, by similar reasoning, generated by characteristic curves $C_{1}$ of $f_{1}=a_{1}$, whose strips again satisfy $p=\phi, q=\psi$.

It follows that at the intersection of a $C$ and a $C_{1}$ the strips corresponding to $C$ and to $C_{1}$ have a common surface element, which must therefore be the element of $S$ at that point (since two intersecting lines determine an element); and as every point of $S$ is an intersection of a $C$ and a $C_{1}, S$ must be an integral surface of $f=0$ and $f_{1}=a_{1}$ (and also of $f_{2}=a_{2}$ since the argument is quite symmetrical with respect to $f_{1}$ and $f_{2}$ ).

Conversely, if $S$ is an integral surface of $f=0$, we can show that $\left[f_{2}, f_{1}\right]=0$.

For then the normal at any point of $S$ is given by $p=\phi, q=\psi$ (since the strips belonging to the curves $C$ now lie on the surface $S$ ). Hence $S$ is also an integral surface of $f_{1}=a_{1}$ and so is generated by 
characteristic strips of $f_{1}=a_{1}$. Let $f_{3}=a_{3}, f_{4}=a_{4}$ be the other two independent integrals of $\left[F, f_{1}\right]=0$. Then

$$
\left.\begin{array}{l}
f_{3}(x, y, z, \phi, \psi)=a_{3} \\
f_{4}(x, y, z, \phi, \psi)=a_{4}
\end{array}\right\}
$$

together give the congruence of characteristic curves of $\left[F, f_{1}\right]=0$ whose associated strips satisfy $p=\phi, q=\psi$.

If $\lambda\left(a_{3}, a_{4}\right)=$ const., then these curves generate the surface $\lambda\left(f_{3}, f_{4}\right)=$ const., $\lambda$ being an arbitrary function. By choosing $\lambda$ suitably, they can be made to generate $f_{2}(x, y, z, \phi, \psi)=a_{2}$, since we know that this is generated by curves of the above congruence; $f_{2}$ must then be of the form $\lambda\left(f_{3}, f_{4}\right)$, and consequently must satisfy $\left[F, f_{1}\right]=0$.

The condition $\left[f_{1}, f_{2}\right]=0$ is thus both necessary and sufficient. 